\title{
HIGH CIRCULATING LEVELS OF SICAM-1 AND SVCAM-1 IN PATIENTS WITH HASHIMOTO THYROIDITIS
}

Aysenur Ozderya, MD ${ }^{1}$, Kadriye Aydin, $\mathrm{MD}^{1}$, Sule Temizkan, $\mathrm{MD}^{1}$, Semra DogruAbbasoglu, $\mathrm{MD}^{2}$,Pervin Vural, $\mathrm{MD}^{2}$, Yuksel Altuntas, $\mathrm{MD}^{3}$

${ }^{1}$ Div of Endocrinology, Dr.Lutfi Kirdar Kartal Training and Research Hospital, Istanbul, Turkey,

${ }^{2}$ Department of Biochemistry, Istanbul Faculty of Medicine, Istanbul University, Capa, Istanbul, Turkey

${ }^{3}$ Department of Endocrinology, Sisli Etfal Training and Research Hospital, İstanbul, Turkey

Objective: Elevated levels of the soluble endothelial adhesion molecules, sICAM-1 and sVCAM-1 reflect chronic inflammatory state. Inceased levels of sICAM-1 and sVCAM-1 were demonstrated in various autoimmune thyroid diseases with abnormal thyroid function, but their clinical significance is undefined, especially in the euthyroid patients with Hashimoto's thyroiditis (HT). Increased levels of soluble adhesion molecules are also associated with components of the metabolic syndrome like diabetes mellitus, insulin resistance and obesity, and beyond thyroid dysfunction, HT is believed to affect metabolic parameters. Accordingly, in the present study we aimed to analyze sICAM- 1 and sVCAM-1 levels in HT, their relation with thyroid autoimmunity and glucose metabolism markers in HT.

Methods: Eighty patients with HT, and age- and BMI-matched 80 control participants were enrolled. Serum sICAM-1, sVCAM-1, free triiodothyronine (fT3), free thyroxine (fT4), thyroid-stimulating hormone (TSH), thyroid peroxidase antibody (anti-TPO), thyroglubulin antibody (anti-TG), fasting blood glucose, insulin, lipid levels and homeostatis model assessment for insulin resistance (HOMA-IR) were assessed in all participants.

Results: Patients with HT had significantly higher levels of sICAM-1 and sVCAM-1 than controls (both $\mathrm{p}<0.001$ ) as well as glucose metabolism parameters. Correlation analysis revealed that both sICAM-1 and SVCAM-1 were significantly positively correlated with $\mathrm{TSH}$, glucose, anti-TG and antiTPO; while sVCAM-1 was correlated with fasting insulin, HOMA-IR, HDL-C positively and with TG negatively in all participants but not in HT patients and control group separately. Regression analysis demonstrated that SICAM-1 was related with anti-TPO and SVCAM-1 was related with both antiTPO and anti-TG.

Conclusion: sICAM-1 and sVCAM-1 levels were significantly elevated in HT and correlated closely with thyroid autoimmunity. Moreover, soluble adhesion molecules, specifically sVCAM-1 had a strong relation with metabolic parameters in HT. 